\title{
Situación del patrimonio moderno en Lima Análisis de casos
}

\begin{abstract}
María Alejandra Bustamante Pacheco / Juan Marco Ureta Córdova / Pamela Carolina Hung Arrunátegui / Ana Belén Scudere Cárdenas / Cynthia Estefanía Florián Holguín
\end{abstract}

Universidad de Lima, Perú Recibido: 3 de diciembre de 2015 / Aprobado: 2 de febrero de 2016

El presente artículo fue desarrollado en conjunto por un grupo de alumnos del curso Conservación del Patrimonio Edificado, durante el año 2015. Metodológicamente, los estudiantes revisaron las publicaciones de la revista El Arquitecto Peruano desde el año 1947 hasta 1960, con la intención de encontrar edificios emblemáticos que puedan contrastarse con la situación en la que se encuentran hoy. Esto derivó en un taller de registro que finalmente fue consignado en los documentos que a continuación presentamos.

patrimonio moderno, conservación, centro histórico, arquitectura

\section{Condition of Modern Heritage in Lima. Case Analysis}

This article was jointly developed by a group of students within the course of Built Heritage Conservation during 2015. Methodologically, students reviewed several publications of The Peruvian Architect magazine, dated from 1947 to 1960 with the intention of finding landmark buildings that may be contrasted with their current conditions. This resulted into a registration workshop which was finally recorded in the documents presented below.

modern heritage, conservation, historic center, architecture 


\section{LA CASA HUIRACOCHA}

Fue diseñada por el arquitecto Luis Miró Quesada $^{1}$ en el año 1947 y terminada de construir en 1948, tras una larga lucha con el municipio por el trámite de los permisos y autorizaciones del caso. En esos años, la normativa exigía que ninguna casa cercana a esa zona tuviera un diseño que no fuera neocolonial. Luego de un año de insistencia, al fin esta licencia de construcción fue otorgada y se inició la obra.

El arquitecto Miró Quesada buscaba no solo innovar con una arquitectura que en el Perú no se veía comúnmente, sino también crear espacios únicos que tuvieran facultades propias de la arquitectura moderna, que aún no se exploraba en el ámbito local. Esto lo lograría con la búsqueda, en el proyecto, de un espacio interior que debe experimentarse con fluidez y dinamismo y no como espacios fragmentados; sin embargo, este espacio, con su propia forma, controlaría las visuales. El único límite del gran espacio serían las visuales controladas por el diseño (Miró Quesada, 1987, p. 74).

Otro punto importante del partido era la privacidad con el entorno. Esto se logró direccionando los frentes de las visuales hacia un jardín y resolviendo el espacio de servicio al disponer su ubicación en un extremo del lote y diferenciarlo de los niveles de la Casa con un medio nivel con respecto al área

1 Luis Miró Quesada (Lima, 1914-1994) fue un destacado arquitecto peruano, docente, periodista, crítico de arte, ensayista y sobre todo un gran promotor del arte moderno en el Perú. También fue catedrático en la Universidad Nacional de Ingeniería. social. Luis Miró Quesada también consideró importante el uso del techo como un área útil para la familia, y decidió zonificar de tal manera que el área con mayor asoleamiento fuera para actividades infantiles o de reunión familiar y la de menor asoleamiento para menesteres de servicio.

Por último, vio que el entorno que los rodeaba podía ser aprovechable con vistas, e hizo un balcón corrido con visuales a estos lugares y techado para controlar el asoleamiento.

Es importante identificar las influencias y entender las circunstancias de la construcción de la Casa, que fue proyectada en 1947 y terminada de construir a mediados de 1948. La Casa se descubre como un producto cultural que busca alcanzar una versión de modernidad peruana y universal en un contexto de transformación social y búsqueda de una nueva identidad moderna. Esta obra se propone como catalizador de una renovación arquitectónica y de una transformación cultural capaz de responder a las demandas de una nueva época. La Casa sintetiza el nuevo cambio que experimenta nuestra arquitectura hacia la aceptación de lo moderno, lo que se expresa en el uso de la planta libre, en la integración de los espacios interiores, en la independencia de la estructura respecto a los muros o curvas, así como en una gran libertad en el uso de las ventanas; todo ello, dentro de una nueva forma del concepto de la racionalidad espacial y constructiva.

La Casa Huiracocha ha sido constantemente intervenida a lo largo de los años, y ha sufrido cambios a manos de su mismo arquitecto mientras tuvo vida y más tarde por parte de los 
nuevos propietarios. Es muy cierto que algunas modificaciones son necesarias para mejorar las instalaciones o repararlas cuando ya no tienen la misma eficiencia de antes, pero cuando estos cambios se realizan por factores externos a la conservación se tornan contraproducentes $\mathrm{y}$ terminan deteriorando la arquitectura $\mathrm{y}$ quitándole el sentido histórico, llegando a transformar una obra casi por completo con tan solo un cambio mínimo pero esencial para su conservación.

Con el tiempo cambiaron algunos materiales, como la baranda exterior del balcón, que en un inicio estaba hecha de láminas onduladas, posteriormente reemplazada por una baranda compuesta de planos horizontales y perfiles metálicos que ha cambiado la materialidad original de la casa. Se trata de una forma de "modernización" que no busca conservar el bien y disminuye su valor constructivo.

La fachada no es el único elemento modificado. En el interior, el primer cambio ocurrió cuando el arquitecto decidió hacer una pequeña ampliación de su casa en el jardín posterior. Con la muerte del arquitecto la familia decidió vender la propiedad, y con el nuevo dueño se impuso un cambio en la destinación de uso del inmueble. Hoy en día los espacios interiores se han perdido, el gran espacio que creó el arquitecto se ha visto fragmentado por tabiquería destinada a generar oficinas y se perdió esa relación de espacio fluido y controles visuales de la espacialidad original.

La seguridad de la Casa también ha supuesto el añadido de un elemento de control, con la ingenua intención de "conservar" la fachada a la vista de todos los peatones, y se decidió cerrar el frente mediante una reja que limita la propiedad del área pública. Estos cambios pueden ser interiores o de fachada, pero también afectan la materialidad del inmueble. En un inicio, la Casa fue planteada con un aparejo expuesto de ladrillos y buscando lograr esa sinceridad constructiva que dejaba sin revestimientos sus materiales. Con el pasar del tiempo se fue perdiendo este criterio, los ladrillos expuestos han sido pintados de diferentes colores (incluso en color "ladrillo") y ha desaparecido una característica vital de la modernidad de la Casa.

Esta obra tiene una gran importancia, por su valencia histórica marca un hito en la arquitectura en el Perú, y por ello se debería proteger de mejor manera, no solo manteniéndose viva la obra sino también aplicando ciertos criterios de compatibilidad de la nueva función, que debe ser acorde con los valores espaciales y constructivos de la misma. Lamentablemente, la Casa Huiracocha está siendo modificada por dentro para cumplir con nuevas necesidades, sin tenerse en cuenta un criterio conservador, de mínima intervención, de preservación de la originalidad del inmueble y para evitar el cambio de la misma naturaleza de la obra, sin agregarle elementos que la modifiquen, como sucede en este caso con los añadidos de la reja perimetral, el cambio en el parapeto del balcón y el color de la casa.

Si no evaluamos lo urgente de una valoración de lo que poseemos como colectividad, aquello que aún sobrevive en precarias condiciones dejará de existir y terminaremos lamentando el no haber protegido este tipo de obras, 


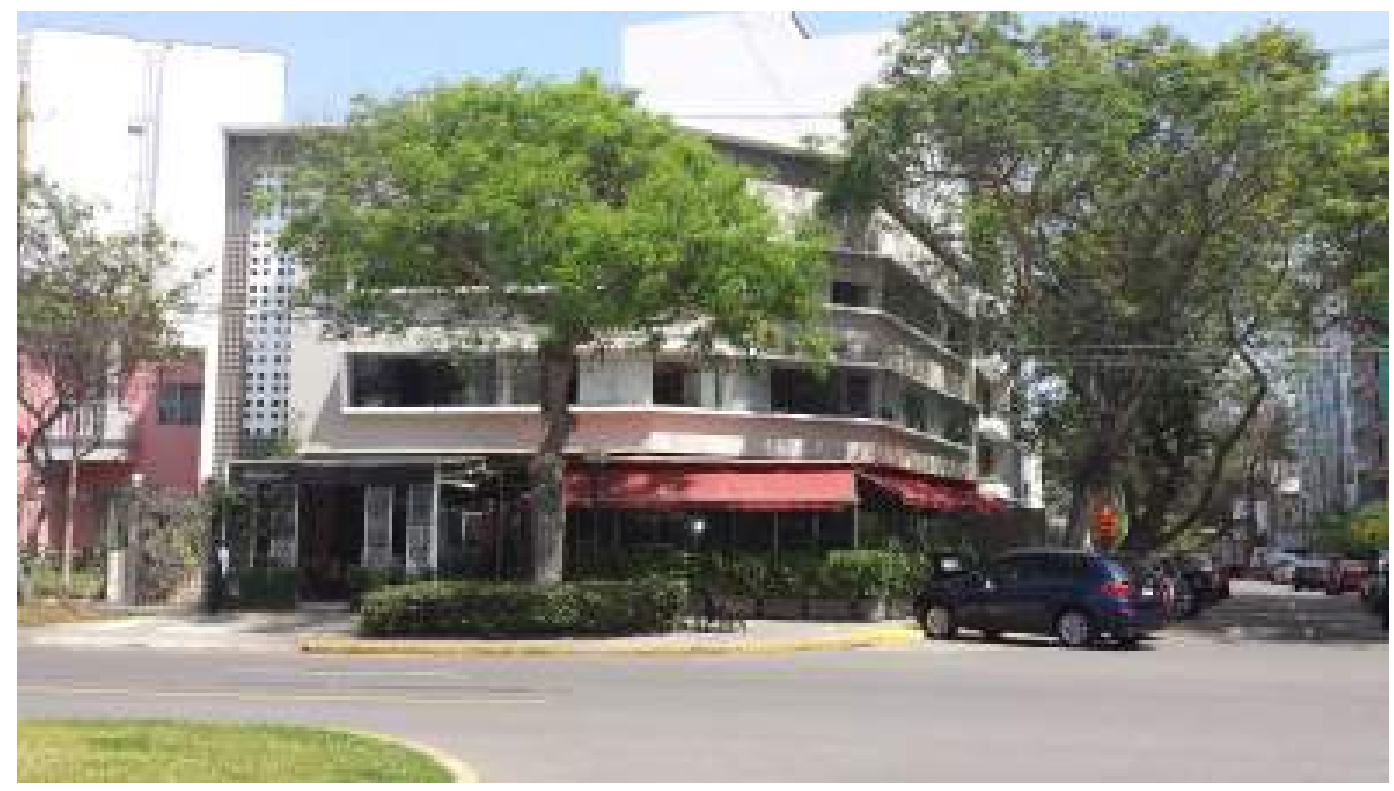

Figura 1.

Edificio San Fernando, 2015

Fuente: Fotografía de Martín Fabbri

símbolos de cambio y parte de la historia de la arquitectura peruana.

\section{EDIFICIO SAN FERNANDO}

En la actualidad, muchos edificios antiguos, entre ellos algunos históricos, quedan en el abandono y por consiguiente son demolidos con el paso del tiempo; ya que no les encontraron un adecuado uso que a su vez fuera compatible con las necesidades de los usuarios durante la vida de los edificios y proyectos. Pero existen distintas maneras de conservar edificios históricos, a fin de prolongar y mantener el mayor tiempo posible los materiales de los que están constituidos. Este objetivo puede realizarse mediante intervenciones de "conservación preventiva o indirecta", esto es que no actúan sobre la estructura física del objeto; o bien mediante la "intervención directa de conservación", que se ejerce sobre la estructura física del objeto cultural (Gonzales Varas, 2005, p. 539). En este caso se adaptó el uso de oficina a la estructura original de una vivienda. En Lima, este método se emplea desde hace unos veinte años, en los distritos más céntricos, como son San Isidro y Miraflores.

El proyecto Edificio San Fernando, obra del año 1951, fue diseñado por el arquitecto Aramburú 
Menchaca $^{2}$, graduado de Arquitectura e Ingeniería de la Universidad Nacional de Ingeniería y de trayectoria destacada por obras como la Vía Expresa del Paseo de la República (1966), el Proyecto de la Costa Verde (1968), las Ciudades Satélites de Ciudad de Dios, Ventanilla, Puente Piedra y Vitarte; entre otras.

Este proyecto de tres pisos está emplazado en una esquina, y es un proyecto de vivienda. El arquitecto planteó que la planta baja estuviera destinada exclusivamente al comercio y para este fin se retiró el frente del proyecto un metro con respecto a la fachada del segundo nivel. Existen dos ingresos a las viviendas, por las dos caras de la esquina. El comercio tiene una continuidad con el nivel de calle, mientras que los ingresos a la vivienda tienen un cambio de nivel de cuatro gradas. El segundo y el tercer nivel están distribuidos en dos departamentos por piso y con uso común de la azotea. La fachada del edificio está inclinada unos treinta grados hacia adelante, lo que le da una perspectiva dinámica y muy singular.

Hoy en día, luego de más de medio siglo de construido, el edificio se conserva prácticamente tal cual. El mayor cambio que ha tenido es el de su uso, ahora como lugar de oficinas sobre todo, por estar en un distrito tan céntrico como Miraflores. Esta modificación resulta razonable para la conservación del edificio. Por otro lado, una modificación fisica que ha sufrido es la del

2 Ernesto Aramburú Menchaca (Lima, 1920-2010) fue un distinguido arquitecto peruano, alcalde de Miraflores, director de obras, teniente alcalde y concejal en los municipios de Lima Metropolitana, Miraflores, Jesús María y Pueblo Libre. Además, creador del circuito de playas de la Costa Verde y generador de la recuperación del Parque Reducto y de proyectos de planificación urbana de Lima. primer piso, que originalmente estaba retirado con respecto al segundo. Ahora que existen dos restaurantes en el primer nivel, se ha ocupado el retiro con elementos vidriados y jardineras. Aunque se trata de una variación importante, tiene la virtud de ser reversible y no afectar de manera drástica al edificio.

Este proyecto es un ejemplo claro y sencillo de que la adaptación a otros usos de un edificio histórico, para su conservación y vigencia en el tiempo, puede funcionar de manera correcta y ser armoniosa con el edificio y su entorno. Como este, hay muchos otros proyectos que se pueden tomar como ejemplos, con el fin de evitar lo que viene sucediendo con nuestro patrimonio arquitectónico, el cual, poco a poco, se deteriora y desaparece.

\section{EDIFICIO LIMATAMBO. SIN LUGAR PARA LA MEMORIA}

En la segunda mitad del año 2013 se inició el desmantelamiento del emblemático edificio Limatambo, ubicado en la intersección de la avenida Javier Prado con la Vía Expresa. El retiro del letrero circular que por muchos años coronó su azotea anunciaba su inminente desaparición, que muchos profesionales y periodistas quisieron detener a través de exhortaciones a organismos gubernamentales como el Ministerio de Cultura, donde una recién nombrada ministra Diana Álvarez Calderón inclinaba la balanza en favor de la inversión privada y hacía recordar cómo su antecesora, Cecilia Bakula, en el año 2008, como directora del INC ${ }^{3}$, avalaba la demolición

3 Sigla del Instituto Nacional de Cultura del Perú, anterior dependencia del Ministerio de Educación para asuntos 


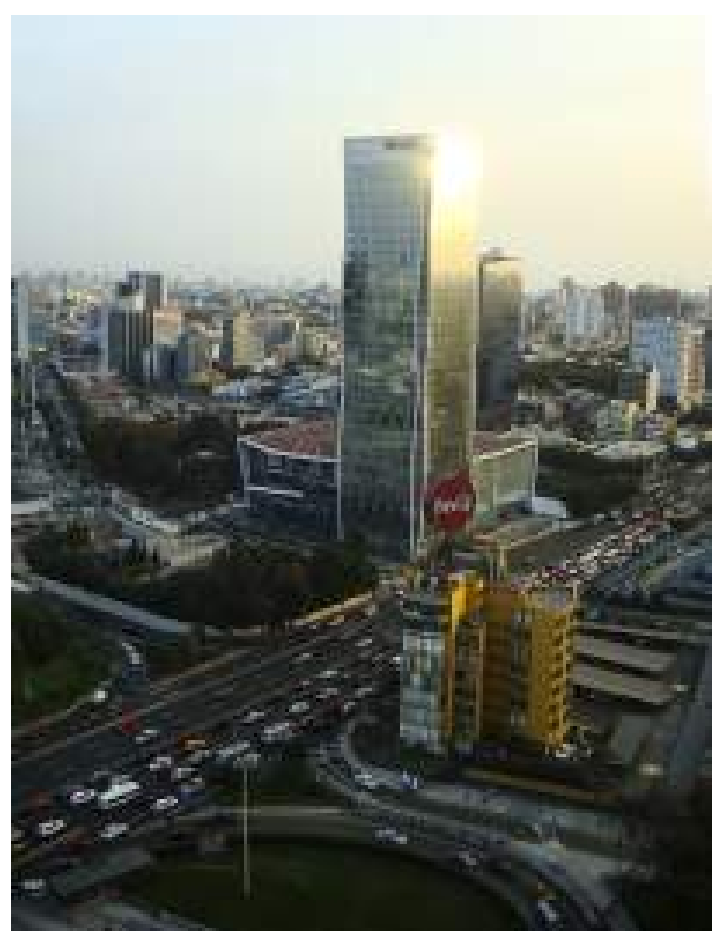

Figuras 2 y 3 .

Edificio Limatambo antes y después de la demolición Fuente: Fotografías de Pamela Hung

del Santuario del Señor de Luren por verse afectado por el terremoto del 15 de agosto del 2007, sin evaluar alternativas de restauración que mantuvieran su identidad e historia. Felizmente, la presión del pueblo iqueño logró detener la demolición total del templo.

Con el edificio Limatambo no sucedió lo mismo. Personas comprometidas con estos valores acudieron a organismos culturales para solicitar su apoyo, a fin de preservar

patrimoniales. Sus funciones han sido asumidas hoy por el Ministerio de Cultura.

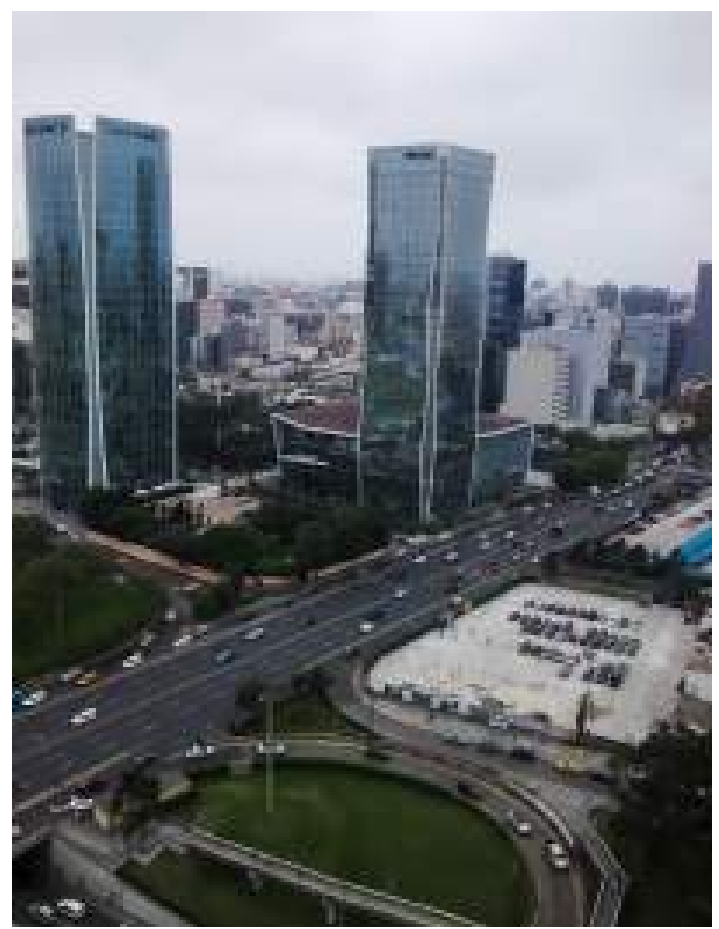

la identidad de nuestra ciudad y evitar que un edificio que era parte de la historia de la arquitectura moderna limeña desapareciera para dar paso al crecimiento comercial impulsado por una familia dedicada por años a los negocios inmobiliarios. Sin embargo, se dieron con la sorpresa de que para tomar acciones legales este edificio tendría que haberse declarado como Patrimonio Histórico y no lo fue. Esta coyuntura aceleró aún más el proceso de desmantelamiento, evitando que algunos artistas pudieran realizar las últimas sesiones fotográficas del edificio - a manera de preservar un registro dentro de la memoria de la ciudad- e impidiendo una marcha atrás en 
las decisiones de las autoridades que ya habían otorgado la licencia de demolición. Ayudó en su momento que las opiniones se encontraran divididas entre los propios arquitectos.

Para las jóvenes generaciones, el edificio Limatambo, opacado por el crecimiento desbordado de Lima y las construcciones aledañas, es poco más que un pedestal para el panel giratorio de Coca-Cola. Para quienes presenciaron de primera manola transformación de la ciudad, la caída del otrora gigante solitario manifiesta la carencia de un criterio elemental por parte del Ministerio de Cultura para declarar patrimonio arquitectónico aquello que merece ser protegido. (S/a, 2013)

Los arquitectos José García Bryce ${ }^{4}$ y José Bentín ${ }^{5}$ se pronuncian acerca de una polémica que incluso ha repercutido en las redes sociales. García Bryce, quien comenzó su carrera en las oficinas de Seoane, asegura:

No existen los mecanismos de tipo reglamentario que permitan conservar estos ejemplos de patrimonio arquitectónico. No es el único edificio valioso que ha sido demolido, y probablemente no será el último. Es algo negativo desde el punto de vista de la

4 Arquitecto y catedrático peruano (Lima, noviembre de 1928), destacado por ganar el Hexágono de Oro en 1981; Premio Nacional de Fomento de la Cultura, área Arquitectura (Premio Chavín) en 1963. Obras: Conjunto Habitacional Chabuca Granda, Capilla San José en la Urbanización Apolo en La Victoria, etc.

5 Arquitecto peruano (Lima, 1938-2015), profesor de la Universidad Nacional de Ingeniería y miembro honorario del Colegio de Arquitectos del Perú. Obras: Conjunto Habitacional Los Próceres en Santiago de Surco; sede de SENCICO en San Borja; Instituto Nacional de Salud del Niño en San Borja; remodelación y ampliación del Estadio Nacional en Lima; Centro de Alto Rendimiento de la Villa Deportiva Nacional VIDENA de San Luis; y la sede del Banco Surmebanc en San Isidro. historia de la arquitectura moderna del Perú. (S/a, 2013)

Para el arquitecto José Bentín, en cambio, "no hay que ser más papistas que el Papa". Bentín asegura que es el aislamiento del edificio Limatambo el que lo condena a desaparecer, "cosa que sería impensable en el caso del edificio Diagonal, también de Seoane, ubicado frente al Parque Kennedy y totalmente asimilado al entorno" (S/a, 2013).

Ya la suerte está echada para una estructura diseñada por uno de nuestros grandes arquitectos, Enrique Seoane Ros ${ }^{6}$, estructura que se convirtió en un símbolo de la historia del desarrollo urbano de Lima contemporánea, sin opciones a barajar alternativas de recuperación. Lo más desalentador es que el grupo Brescia, propietario del edificio, tuvo en sus manos la posibilidad de dar una clara señal de compromiso con la ciudad y no lo hizo. Como sostiene Joan Closa Pujabet, jefe del Servicio de Patrimonio Arquitectónico de la Provincia de Barcelona:

La identidad no pasa por cuestiones nacionalistas. Pasa por el arraigo sentimental: por la iglesia donde mis padres se casaron, donde bautizaron a mi abuelo, un edificio que he visto toda mi vida. Esa es la identidad. Ese es el patrimonio. El valor emocional de los lugares, de los edificios, tiene que ser tomado en cuenta. (Como se cita en Lizarzaburu, 2012, p. 12)

6 Arquitecto peruano (Lima, 1915-1980). Sus obras abarcaron desde viviendas unifamiliares de la alta sociedad, hasta edificios públicos y privados. Entre ellas se encuentra el edificio Rizo Patrón en Lima, el Tacna-Nazarenas en Lima, el edificio Wilson en Lima, el Ministerio de Educación en Lima, el edificio Diagonal en Miraflores, Residencial Limatambo en San Isidro, el edificio del Banco Wiese en Lima, entre otras. 
La representativa obra de Enrique Seoane fue demolida para dar lugar a una torre que muestra que el progreso y la memoria no siempre van de la mano. Las posiciones son encontradas. En el artículo "Edificio Limatambo: sin lugar para la memoria" (S/a, 2013), Bentín recuerda el caso de la Casa Marsano, en la avenida Arequipa, que fue demolida en 2002 ante la negativa del INC de declararla patrimonio monumental. Poco pudieron hacer los vecinos miraflorinos que salieron a la calle para evitar ese derrumbe (hasta el momento, las tibias protestas por la caída del edificio Limatambo se dan solo en plataformas virtuales). En esa ocasión, la Casa Marsano cayó porque se edificaría en su lugar una lujosa torre de la cadena de hoteles Hilton. Hoy, en su lugar, no se eleva ningún lujoso hotel, sino el centro comercial Compu Palace, sin ninguna calidad arquitectónica, o como dijeron algunos, la "estética de lo barato".

En el caso del edificio Limatambo, hoy ya demolido, se planea construir un edificio con más de cuarenta pisos de altura, el primer rascacielos del Perú, tratando de concentrar los edificios representativos ya construidos, como son hoy en día el Hotel Westin y la sede del banco GNB (anteriormente sería el HBSC), diseños pertenecientes al estudio Fort-Brescia.

Uno de los comentarios que se repite en las redes es que se está priorizando el mercado en detrimento de la herencia histórica. Y es cierto que es el sector privado el que parece decidir, adaptando las normativas municipales, qué y cómo se construye en Lima. A pesar de sus diferencias con respecto a la importancia histórica del edificio Limatambo, tanto Bentín como García Bryce están de acuerdo en la debilidad de las municipalidades frente a los grupos de poder. "Los reglamentos no son coherentes”, afirma García Bryce en el artículo ya citado, "la zonificación y las alturas cambian constantemente por presión de las inmobiliarias y los propietarios, pero no de acuerdo con lo que le conviene a la ciudad". Para Bentín, los planes urbanos municipales dejan mucho que desear:"Se preocupan por la zonificación, pero sin dar normas que tengan que ver con la continuidad de la ciudad", manifiesta. El papel de la inversión privada en el desarrollo de la urbe es fundamental, pero debe ir de la mano de políticas municipales sólidas y coherentes. Si no se puede conseguir eso, menos se podrá hablar de proteger el patrimonio arquitectónico.

Como sugiere José García Bryce, no se trata de ir por la vena de "todo tiempo pasado fue mejor", pero sí de complementar progreso y memoria, herencia y porvenir. "El Perú está atrasado en ese sentido”, afirma, “... no en comparación con países europeos o norteamericanos, sino con otros países latinoamericanos que ya tienen catalogadas como monumentos sus obras modernas. Nosotros no tenemos eso, y es nuestro déficit". Tampoco se establecen normas que busquen un equilibrio entre el estado original de la edificación y la propuesta de remodelación; que analicen por ejemplo la espacialidad, el paisajismo, el contexto histórico, tecnológico y ecológico, fomentando un círculo virtuoso que ayude a mantener en buen estado las edificaciones consideradas patrimoniales.

Sin embargo, podemos concluir que hoy nos desempeñamos dentro de un círculo vicioso que se caracteriza por la indolencia, el abandono y deterioro de la construcción, que culmina con la inevitable demolición del inmueble por su estado de total precariedad, como fue el caso del famoso solar "El Buque" en Barrios Altos, que siendo catalogado 
como patrimonio histórico por ser el primer complejo de departamentos de América, con una historia que data desde antes de la guerra con Chile, pasando por las famosas reuniones festivas de los criollos de antaño, se tuvo que demoler en su integridad debido a un incendio provocado por sus deficientes instalaciones eléctricas, cuando inclusive la acción interventora de los bomberos terminó de dañar lo poco que quedaba de esta edificación, que se desplomó en su totalidad.

Dentro de ese contexto de indiferencia, los que hoy nos sentimos comprometidos con nuestro patrimonio tenemos el compromiso de salvaguardarlo con políticas que realmente fomenten y ayuden a preservar con éxito los edificios históricos de nuestro país. Tal cosa no es algo imposible. En la ciudad de Buenos Aires, los edificios antiguos han sido conservados e intervenidos en algunos casos, añadiéndoles un toque de modernidad pero sin alterar su estilo y concepto, lo que ha permitido que no se pierda el aspecto comercial y rentable del lugar.

El exedificio Limatambo quedará en nuestra memoria como el recuerdo de un edificio que pudo integrarse a una propuesta arquitectónica moderna, como alternativa de convivencia del pasado con el presente, pero que sucumbió ante un vacío de normatividad, ante la indiferencia de las autoridades y el pragmatismo de las constructoras.

\section{EDIFICIO NYCI PARA INVERSIONES INMOBILIARIAS ${ }^{7}$}

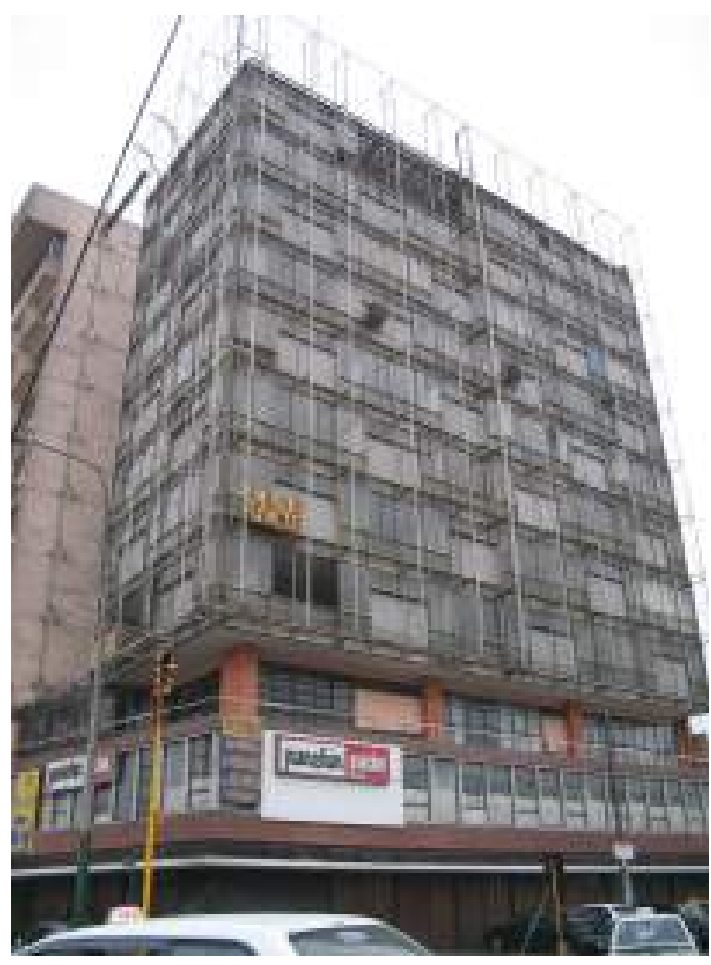

Figura 4.

Edificio Nyci

Fuente: Fotografía de Ana Belén Scudere

Este nuevo edificio, situado en la esquina del Paseo de la República y la avenida Raimondi, en Lima, favorablemente enmarcado por las áreas verdes que lo rodean, ha contribuido sin duda a dar realce a esta zona de la ciudad que poco a poco va cobrando un auge comercial. La base del edificio, conformada por el primer piso y la mezzanine, ocupa

7 La información técnica del edificio ha sido compartida en Rondinel y Vigil (2007). 
la totalidad del terreno. La mezzanine, con su volumen volado en todo el perímetro interior, determina de esta manera una altura de cielo de siete metros aproximadamente, lo que crea un ambiente espacioso y aireado, destinado para el funcionamiento de locales comerciales. (S/a, 1958, pp. 27-29)

Por otro lado, en los pisos superiores estaban los consultorios médicos y la residencia particular del arquitecto.

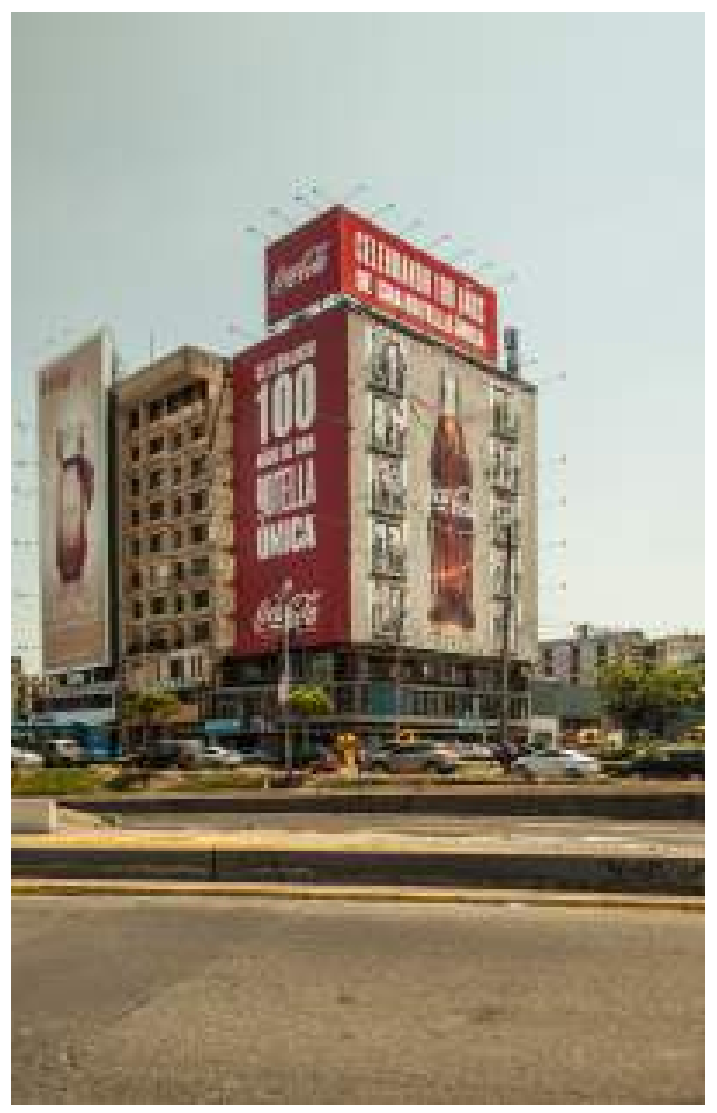

Figura 5.

Edificio Nyci

Fuente: Fotografía de Martín Fabbri
El cambio que ha sufrido el edificio de Seoane es evidente, pero hay grandes rasgos que con el tiempo se han ido perdiendo. El rasgo más resaltante que se consigna en el artículo citado anteriormente es el tratamiento exterior del edificio, que de concreto armado, bastidores de aluminio y paneles tratados en color azulado, se ha transformado en un cartel de publicidad que cubre completamente el inmueble. Es la negación absoluta de la arquitectura. Al ser recubierto con este elemento, el edificio perdió su esbeltez y permeabilidad, que se lograba con ese fuerte espacio libre que dejaba entre la zona comercial y la vivienda. Esta estructura metálica que cubre absolutamente todo el proyecto solo contribuyó a degradarlo, de tal forma que los tres cuerpos que Seoane había marcado se perdieron completamente, haciendo que pase desapercibido y que se convirtiera en "otro edificio con un cartel en Lima".

Otro cambio importante es el que sufrió el contexto en donde se ubica el edificio. En las fotografías podemos ver cómo resalta el proyecto y lo imponente que se ve gracias a ese marco de árboles que tiene, y lo solitario que se encontraba; sin embargo, debido al desarrollo urbano posterior de la urbe, Lima sufrió varias modificaciones, como la avenida Paseo de la República. Dicho cambio se ve reflejado con la perspectiva de la fotografía, ya que nos podemos dar cuenta de que esta calle tenía un flujo bajo de autos, lo cual permitía cruzar a los peatones e incluso tomar una fotografia del lugar. Hoy, la hazaña de atravesar ese tránsito es realizada por pocos. Por otro lado, está la contaminación visual debida a los dos edificios de altura similar ubicados en sus flancos, que saturan el paisaje urbano, además de que aquellos adoptaron la peor característica del actual edificio, la publicidad en la fachada. 
Aparte de ser el proyecto de un arquitecto peruano reconocido, como Seoane, los últimos tres pisos eran su residencia particular: en el noveno piso estaban los dormitorios, en el décimo la recepción y los servicios, y en el decimoprimero estaba su estudio, el espacio más importante, ahí donde creaba sus proyectos, donde diseñaba y pensaba horas de horas, entre esas cuatro paredes que ahora son apenas un espacio sucio y olvidado.

Finalmente, las fotos presentadas en este artículo fueron tomadas en 2004 (Rondinel \& Vigil, 2007) y muestran el carácter de abandono en el que se encuentra el edificio. El exterior queda en un segundo plano y los inquilinos del edificio revelan poco interés por lo que sucede en la fachada. Es así que debemos preguntarnos cuánto tiempo va a pasar hasta que los peruanos tomemos conciencia de la necesidad de conservar nuestros edificios emblemáticos -no solo por este caso sino también por las diversas casas, equipamientos, multifamiliares y cualquier otro proyecto de arquitectos reconocidos que hayan construido en nuestro país- y de mantener siempre la actitud de supervisar que los nuevos usos que se les están agregando sean compatibles con su valor histórico.

\section{EDIFICIO DE SEGUROS EL SOL}

Mucho se habla y escribe en la actualidad sobre nuestra "Lima que se va", y abundan las mociones y propuestas para conservar, aun parcialmente, algunas de sus casas y calles tradicionales de mayor valor, pero no son muchos los que se preocupan con la misma intensidad por los edificios y trazos urbanos de la Lima que está llegando. Por ello es que saludamos la erección, justamente en la céntrica Colmena, de otro edificio diseñado por Enrique Seoane, cuyos proyectos contrastan en forma distintiva y aleccionadora con tantos otros de la actualidad, de mediocre concepción, que tienden a vulgarizar el aspecto de la nueva Lima naciente. La Lima que viene muy bien puede, y debe, ser tanto y más bella que la antigua, conservando y reconquistando en sus trazos urbanos y arquitectónicos el aire sobriamente elegante y alegremente señorial, hasta ahora característico de la capital. (S/a, 1959, pp. 10-12)

El edificio El Sol, ubicado en el Centro Histórico de Lima, fue diseñado por el arquitecto Enrique Seoane en 1959 para la Compañía de Seguros El Sol. La idea original consistía en el diseño de un hotel, pero luego de una investigación más profunda se concluyó que la mayor rentabilidad podría provenir de la construcción de oficinas y viviendas, por lo que se diseñó un edificio de usos múltiples con un basamento comercial, cuatro niveles siguientes destinados a viviendas y los restantes para oficinas que aseguraban la rentabilidad de la inversión.

Este edificio está estructurado con un sistema de pórticos de acero que funcionan al conjunto de losas de concreto que permitían una rápida construcción del edificio y grandes luces al interior. Tal sistema correspondía a un gran avance constructivo en el Perú, debido a la novedad de los materiales usados y a la velocidad con que la obra pudo terminarse en conjunto con los acabados de primera para la época. Todos estos agentes combinados permitieron que después de cambiado el uso, y el propietario, el edificio poseyera la suficiente flexibilidad para ser transformado y evitarse su abandono (S/a, 1959, pp. 10-12). 
La fachada corresponde a un muro cortina con marcos de aluminio con aplicaciones modulares, que han sido conservados a primera vista. En 2012 fue su último mantenimiento importante, cuando además se cambiaron los paños de vidrio que poseían originalmente, por otros con mayor transparencia, para lograr un mayor ingreso lumínico al interior de las oficinas.

En el año 1977 las acciones de "El Sol Nacional Compañía de Seguros y Reaseguros" fueron compradas en su mayoría por la aseguradora Mapfre (Mapfre, 2008, pp. 7-16). Dentro de las adquisiciones estuvo el edificio en mención, que actualmente funciona como un call center para la compañía. Las plantas han sido modificadas, como se ha mencionado anteriormente, esto se debe al cambio de uso del edificio, en donde el primer nivel está dividido en cubículos de venta para una librería y tiendas de ropa; el segundo nivel también posee un uso comercial, pero al igual que otros edificios del centro de Lima, este no tiene el uso que se destina y por lo tanto se convierte en un gran espacio de almacenes, en este caso para abasto de la librería del primer nivel, hasta que pueda ser alquilado.

Estos dos niveles se encuentran completamente cubiertos por una fachada vidriada sin modulación de aluminio, con losas cubiertas con material sintético oscuro que no ha sido reemplazado durante los mantenimientos del edificio, al igual que el cielorraso de los primeros niveles, que continúa siendo tratado con una mezcla de cemento y arena. Según indican los propietarios de los niveles comerciales, existe una preocupación por mantener una imagen de lo que fue el lugar en un momento glorioso, esto a la mano del costo que implicaría una renovación completa

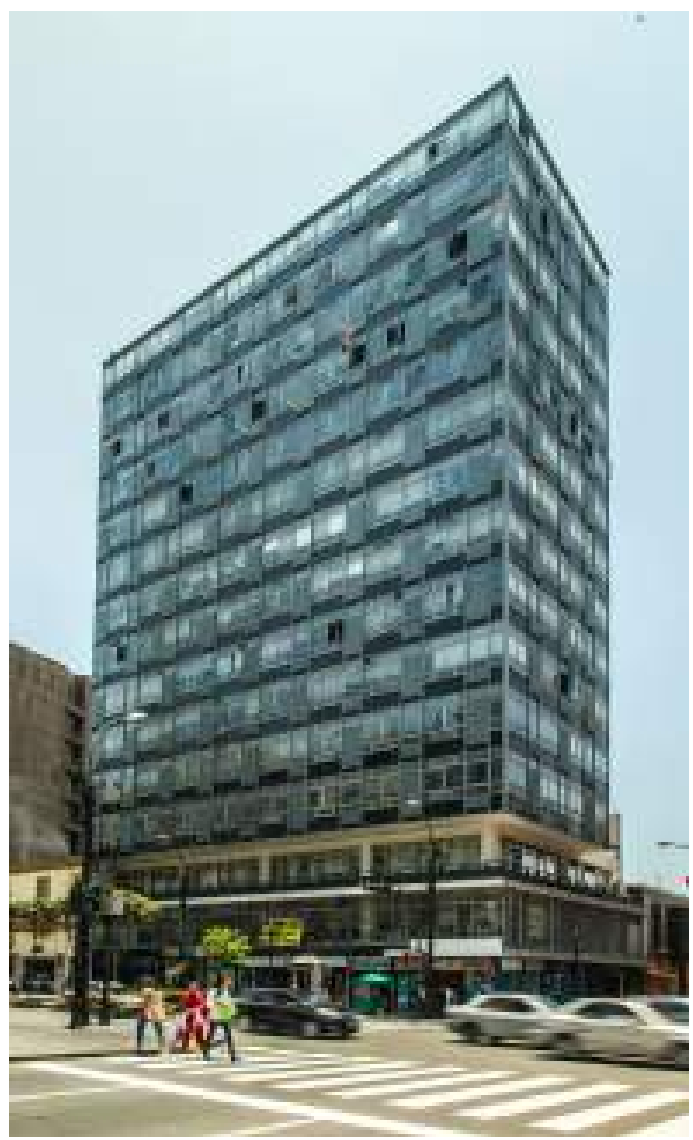

Figura 6.

Edificio El Sol

Fuente: Fotografía de Martín Fabbri

del edificio. Sin embargo, los mayores cambios se dieron en la entrada principal, la cual ha sido modificada para que las personas que trabajan en los niveles superiores puedan ingresar sin ningún problema y mantengan cierto nivel de privacidad con respecto a los trabajadores del basamento comercial. Ambos poseen un mismo escritorio de recepción, que se halla después del recibidor. 
A partir del tercer nivel, el cual está retirado unos metros de la fachada, los pisos han sido casi completamente reestructurados al interior. Ahora existe una separación de cubículos para los trabajadores del call center, donde todo funciona en un único gran espacio. Esto sucede gracias al esqueleto de acero que estructura el edificio, y el cielorraso ha sido modificado para poder cambiar las luminarias por otras más adecuadas para los trabajadores.

El edificio no ha sufrido muchos cambios en cuanto a la fachada se refiere. Esta ha podido mantenerse en sus 56 años de vida, cualidad digna de ser resaltada en una época de transformación constante. La metamorfosis clave de este proyecto se ve en la reestructuración interna, necesaria para evitar el deterioro del edificio por abandono. El cambio le permitió mantener su vigencia a pesar del uso actual, permitido a su vez por el gran diseño estructural y arquitectónico de Seoane, que dotó a la construcción con una flexibilidad adecuada para la época en que vivimos.

\section{REFERENCIAS}

Gonzales Varas, I. (2005). Conservación de bienes culturales: teoría, historia, principios y normas. Madrid: Cátedra.
Lizarzaburu, J. (2012, noviembre 18). La ciudad estaba indignada con la pérdida de sus edificios. El Comercio, p. 12.

Mapfre. (2008). Memoria Anual Mapfre Perú. Lima: autor.

Miró Quesada, L. (1987). Casa Huiracocha. Revista DAU.Documentos de Arquitectura y Urbanismo, 2, pp. 74-75.

Rondinel Oviedo, D. \& Vigil Romero, L. (2007). Reciclaje y renovación urbana de una manzana de La Victoria (tesis de pregrado). Lima: Universidad Ricardo Palma, Facultad de Arquitectura y Urbanismo.

Seoane Ros, E. (1958).Edificio para Inversiones Inmobiliarias S.A. El Arquitecto Peruano, 249-250-251, pp. 27-29.

S/a. (1959). Edificio de Seguros El Sol. El Arquitecto Peruano, 264-265-266, pp. 10-12.

S/a. (agosto, 2013). Sin lugar para la memoria. Recuperado de Habitat. Conservación, reciclaje y restauración: http://www. revistahabitat.com/noticias/val/1466/ sec_val/99/edificio-limatambo-sinlugar-para-la-memoria.html 
